\title{
Fine-Tuning an Algorithm for Semantic Document Clustering Using a Similarity Graph
}

\author{
Lubomir Stanchev \\ Computer Science Department \\ California Polytechnic State University \\ San Luis Obispo, CA 9340\%, USA \\ stanchev@gmail.com
}

\begin{abstract}
In this article, we examine an algorithm for document clustering using a similarity graph. The graph stores words and common phrases from the English language as nodes and it can be used to compute the degree of semantic similarity between any two phrases. One application of the similarity graph is semantic document clustering, that is, grouping documents based on the meaning of the words in them. Since our algorithm for semantic document clustering relies on multiple parameters, we examine how fine-tuning these values affects the quality of the result. Specifically, we use the Reuters- 21578 benchmark, which contains 11, 362 newswire stories that are grouped in 82 categories using human judgment. We apply the $k$-means clustering algorithm to group the documents using a similarity metric that is based on keywords matching and one that uses the similarity graph. We evaluate the results of the clustering algorithms using multiple metrics, such as precision, recall, f-score, entropy, and purity.
\end{abstract}

Keywords: Semantic search; semantic graph; document clustering.

\section{Introduction}

Consider a web portal for an online store. To simplify navigation, merchandise can be grouped into categories. When a new product is introduced, it will be beneficial if the system can automatically classify the product in the correct category. This classification can be performed based on the description of the product. For example, consider a product with the following text description: "white sneakers, size 10". If the system contains the knowledge that the terms "sneakers" and "athletic shoes" are related, then it can classify the new product in the "athletic shoes" category. In this article, we show how such semantic knowledge can be stored in a similarity graph and how it can be used to cluster documents based on the meaning of terms in the documents. We also carefully examine the parameters of the algorithm that builds the similarity graph and the algorithm that performs the clustering. The goal is to fine-tune the two algorithms so that the automatic classification procedure produces results that are as precise as possible.

The problem of semantic document clustering is interesting because it can improve the quality of the clustering result as compared to keywords-matching 
algorithms. For example, an algorithm of the second type will likely put documents that use different terminology to describe the same concept in separate categories. Consider a scientific document that contains the term "ascorbic acid" multiple times and a scientific document that contains the term "vitamin C" multiple times. The documents are semantically similar because "ascorbic acid" and "vitamin C" refer to the same organic compound and therefore the clustering algorithm should take this fact into account. However, this will only happen when the close relationship between the two terms is stored in the system and used during document clustering. The need for a semantic clustering system becomes even more apparent when the number of documents is small or when they are very short. In this case, it is likely that the documents will not share many words in common and a keywords-matching system will struggle to find evidence for grouping documents together. In this article, we go even further by using part of the Reuters-21578 benchmark to optimize the clustering algorithm. This is an important step because we want our algorithm to approximate human judgment as closely as possible.

The problem of semantic document clustering is difficult because it involves some understanding of the meaning of words and phrases and how they relate. Although significant effort has focused on automated natural language processing [9, 10, 24], current approaches fall short of understanding the precise meaning of human text. In fact, we do not know if computers will ever become as fluent as humans in understanding natural language text. In this article, we do not analyze natural language text and break it down into the parts of speech. Instead, we only consider the words and phrases in the documents and use the similarity graph, which is based on a probabilistic model, to compute the distance between pairs of documents. Note that, as described in the next paragraph, most clustering algorithms rely on a distance metric to cluster the documents.

A traditional keywords-matching algorithm for document clustering falls short because it only considers the words and their frequencies in each document. For example, the popular $k$-means clustering algorithm [23] starts with $k$ document seeds. It then finds the documents that are closest to each seed using a distance metric. Next, the centroid (i.e., mean) of each cluster is found and then new clusters are created using the centroids as the seeds. The process repeats and it is guaranteed to converge. The algorithm is based on a vector representation of the documents (based on words frequencies) and a distance metric (e.g., the cosine similarity between two document vectors). Unfortunately, this approach will incorrectly compute the similarity distance between two documents that describe the same concept using different words. It will only consider the common words and their frequencies and it will ignore the meaning of the words. Conversely, our approach adds all the documents to the similarity graph. The distance between a pair of documents is measured by evaluating the paths between them, where a path in the graph can go through several terms that are semantically similar. In this way, we consider not only words, but also phrases (a.k.a. terms) that consist of several words and their meaning. The similarity graph contains directed edges that are labeled with the probabilities that we are 
interested in the destination node given that we are interested in the source node. Note that this implies that the paths in the graph are directed and creating a similarity metric that is symmetric is not trivial and we need to consider paths in both directions.

When computing the similarity distance between two documents, we aggregate the evidence from all the paths between the first and the second document. Every path provides additional evidence about the similarity between the two documents. Note that the weight of a path decreases as the length of the path increases because longer paths provide weaker evidence. Since the paths in the graph are directed, we also examine the paths from the second to the first document and examine how the results can be aggregated. Figure 1 shows the process flow. Creating the similarity graph involves processing information from WordNet about senses, nouns, verbs, and adjectives. Note that words can have many senses and senses can be represented by several words.

We experimentally validate our document clustering algorithm on the Reuters21578 benchmark. Out of the 21,578 newswire stories, 11,362 are categorized in one of several categories using human judgment. Since our algorithm is based on hard clustering, that is, every document can belong to at most one category, we consider only the first human classification for each document. We split the documents between two sets. We use the first set to optimize the parameters of our algorithms. We use the second set of documents for testing purposes. Specifically, we compare the results from the human judgment to applying the $k$-means clustering algorithm with

1. Processing the Senses

2. Processing the Definitions of the Senses

3. Processing the Example Uses of the Senses

4. Processing the Backward Edges

5. Populating the Frequencies of the Senses

6. Processing Structured Knowledge About Nouns

7. Processing Structured Knowledge About Verbs

8. Processing Structured Knowledge About Adjectives

Creating the Similarity Graph

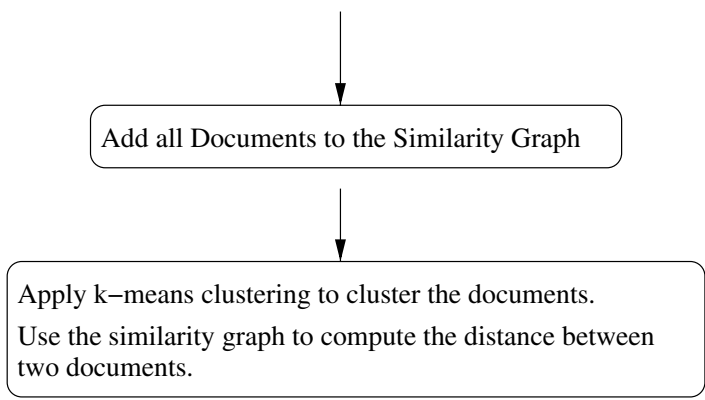

Fig. 1. Process flow diagram. 
two different distance metrics. The first is based on the popular cosine similarity metric that compares two documents as the cosine of the angle between their document vectors. The second metric is based on the distance between the documents in the similarity graph. We use different metrics, such as precision, recall, f-score, entropy, and purity, to evaluate how the results from the clustering algorithms compare to those of human judgment. When we apply the second distance metric, we get results that indicate closer similarity to human classification. This shows that the similarity graph can produce results that more closely match human judgment. The reason is that the similarity graph metric considers the meaning of the words and terms in the documents, while the cosine similarity metric only considers the words and their frequencies. When the parameters of our similarity graph clustering procedure are optimized, our algorithm produces even better results as measured by the different metrics.

In what follows, in Sec. 2 we present a brief overview of related research. The next section describes the steps in creating the similarity graph. Our main contribution in this article is that at each step we look at the parameters that are involved and how they affect the quality of the clustering algorithm. While Sec. 4 explains how to measure the semantic similarity between terms, Sec. 5 describes how to measure the semantic similarity between documents. Again, we consider what parameters are involved and which values produce the best results. Section 6 describes two algorithms for clustering documents: using keywords matching and using the similarity graph. Section 7 validates our semantic clustering algorithm by showing how it can produce data of better quality than the algorithm that is based on simple keywords matching. Lastly, Sec. 8 summarizes the article and outlines areas for future research.

\section{Related Research}

A preliminary version of this article was published in the conference proceedings of the Tenth IEEE International Conference on Semantic Computing [40]. Here, the paper is significantly revised, corrections are made, and more detailed explanations are provided in every section. However, the major contribution of this article is showing how the different parameters of the algorithm that creates the similarity graph and the algorithm that performs the clustering using the similarity graph can be fine-tuned in order to improve the quality of the clustering algorithm.

A plethora of research has been published on using supervised learning models with training sets for document classification [5, 41]. Our approach differs because we use supervised learning only for fine-tuning the algorithm. For example, our original algorithm in [40] is unsupervised, it does not use a training set, and it can cluster documents in any number of classes rather than just classify the documents in preexisting categories.

One alternative to supervised learning is using a knowledgebase that contains information about the relationship between the words and phrases that can be found in the documents to be clustered. For example, in 1986, W. B. Croft proposed the use 
of a thesaurus that contains semantic information, such as what words are synonyms [7]. Sequentially, there have been multiple papers on the use of a thesaurus to represent the semantic relationship between words and phrases [13-15, 17, 19, 27, 32, 42]. This approach, although very progressive for the times, differs from our approach because we consider indirect relationships between words (i.e., relationships along paths of several words). We also do not apply document expansion (i.e., adding the synonyms of the words in a document to the document) when comparing two documents. Instead, we use the similarity graph to compute the distance between two documents. Some limited user interaction is possible when classifying documents - see for example the research on folksonomies [11]. Our system currently does not allow for user interaction when creating the document clusters, but this is an interesting area for future research.

In later years, the research of Croft was extended by creating a graph in the form of a semantic network $[4,30,33]$ and graphs that contain the semantic relationships between words $[1,2,6]$. Later on, Simone Ponzetto and Michael Strube showed how to create a graph that only represents the inheritance of words in WordNet [21,34], while Glen Jeh and Jennifer Widom showed how to approximate the similarity between phrases based on information about the structure of the graph in which they appear [18]. All these approaches differ from our approach because they do not consider the strength of the relationship between the nodes in the graph. In other words, there are no weights that are assigned to the edges in the graph.

Natural language techniques can be used to analyze the text in a document $[16$, $26,36]$. For example, a natural language analyzer may determine that a document talks about animals and words or concepts that can represent an animal can be identified in other documents. As a result, documents that are identified to refer to the same or similar concepts can be classified together. One problem with this approach is that it is computationally expensive. A second problem is that it is not a probabilistic model and therefore it is difficult to be applied towards generating a document similarity metric.

Ontologies can be used for document classification [31]. Our approach is different because we do not consider preselected categories. Using ontologies also requires manual or automatic annotation of each document with a description in a formal language $[12,20,28]$. This may be problematic because manual annotation is time consuming and automatic annotation is not very reliable.

Since the early 1990s, research on LSA (stands for latent semantic analysis [8]) has been prevalent. The approach has the advantage of not relying on external information. Instead, it considers the closeness of words in text documents as proof of their semantic similarity. For example, LSA can be used to detect words that are synonyms [22]. This differs from our approach because we do not consider the closeness of words in a document. Although the LSA approach has its applications, we believe that WordNet provide data of higher quality than the documents to be clustered. 


\section{Creating and Fine-Tuning the Similarity Graph}

In this section, we review how the similarity graph can be created using information from WordNet [25]. The algorithm that creates the graph is previously published in [38]. The novelty is that we use part of the Reuters- 21578 benchmark to fine-tune the algorithm and find the best value for the different parameters.

WordNet gives us information about the words in the English language. The similarity graph is initially constructed using WordNet 3.0, which contains about 150,000 different terms. Both words and phrases can be found in WordNet. For example, "sports utility vehicle" is a term from WordNet. We will sometimes refer to these words and phrases as terms, while WordNet uses the terminology word form. Note that the meaning of a term is not precise. For example, the word "spring" can mean the season after winter, a metal elastic device, or natural flow of ground water, among others. This is the reason why WordNet uses the concept of a sense. For example, earlier in this paragraph we described three different senses of the word "spring". Every term has one or more senses and every sense is represented by one or more terms. A human can usually determine which of the many senses a term represents by the context in which the term appears. WordNet contains about 116,000 senses for the 150,000 terms.

The goal of the similarity graph is to model the relationship between the terms in WordNet using a probabilistic model. For every term that is not a noise word, a node that has the term as a label is created. Similarly, for every sense we create a node with a label that is the description of the sense. All node labels are converted to lower case and we do not create multiple nodes with the same label. We create edges between two nodes with a weight that approximates the probability that someone who is interested in the source node is also interested in the destination node.

\subsection{Processing the senses}

We first show how to build the edges between a term and its senses. Consider the word chair and its three meanings: "a seat for one person", "the position of a professor" and "the officer who presides at meetings". Suppose that WordNet gives a frequency of 35,2 , and 1 , respectively, for the three senses. We will then create the following edges.

$$
\begin{aligned}
& \text { chair } \Rightarrow \text { a seat for one person; weight }=35 / 38 \\
& \text { chair } \Rightarrow \text { the position of a professor } ; \text { weight }=2 / 38 \\
& \text { chair } \Rightarrow \text { the officer who presides at meetings; weight }=1 / 38
\end{aligned}
$$

In general, we will compute the weight of each forward edge as the frequency of the sense divided by the sum of the frequencies of all the senses for the term. The reason is that the term can have many senses and the probability that we are interested in a specific sense depends on the frequency of the sense. 
We will also add backward edges, as shown next.

$$
\begin{aligned}
& \text { a seat for one person } \Rightarrow \text { chair } ; \text { weight }=1 \\
& \text { the position of a professor } \Rightarrow \text { chair } ; \text { weight }=1 \\
& \text { the officer who presides at meetings } \Rightarrow \text { chair } ; \text { weight }=1
\end{aligned}
$$

The weight of each backward edge is always equal to one because there is $100 \%$ probability that someone who is interested in a sense is also interested in the term that represent it. There are no parameters to be set in this step. Note that it is possible that our algorithm creates multiple edges in the same direction between the same two nodes. In this case, we simply add the weights of all the edges and keep a single edge in the final graph. However, this can lead to the weight of an edge being more than one and this is the reason why the weights of the edges are not probabilities in the strict sense.

\subsection{Processing the definitions of the senses}

We next show how to model the relationship between a sense and the non-noise terms in its definition. Note that our algorithm uses a list of about one hundred noise words, such as "who", "where", "at", "about" and so on. Consider the second sense of the word "chair": "the position of a professor". The noise words: "the", "of", and "a" will be ignored. We will therefore be left with two words: "position" and "professor". As a result, we will create the following edges according to the algorithm from [38].

the position of a professor $\Rightarrow$ position; weight $=\operatorname{minMax}\left(0, \alpha_{1}, 1 / 2\right)$

the position of a professor $\Rightarrow$ professor; weight $=0.8 * \operatorname{minMax}\left(0, \alpha_{1}, 1 / 2\right)$

In [38], we assumed that the first words in the definition of a sense are far more important than the later words. We therefore multiplied the edge weight by coef $=$ 1.0 for the first non-noise term and kept decreasing this coefficient by 0.2 for each sequential term until the value of the coefficient reached 0.2 . The function minMax is a custom functions that we will explain later in this section.

Table 1 shows the value for the f-score $(\beta=1)$ with and without using the coef multiplier for different values of $\alpha_{1}$. In this and sequential tables, we will use bold font for the highest value and italic value for the value for the initial algorithm that is described in [38]. Note that the results are for our training set, which includes only the first 2,000 documents in the Reuters collection. The first column in the table shows the results of running the algorithm from [38] and only changing the value for $\alpha_{1}$. The second column shows the result of running the same algorithm for different values of $\alpha_{1}$, but this time we did not multiply by the coef multiplier. In [38], $\alpha_{1}=0.6$ is used, which the table shows to be close to optimal. However, the training data shows that our assumption that the first words in the definition of a sense are more important is incorrect for this application. 
Table 1. The value for the f-score for different values of $\alpha_{1}$ with and without using the coef multiplier.

\begin{tabular}{|c|c|c|}
\hline$\alpha_{1}$ & Using coef multiplier & Without using coef multiplier \\
\hline 0.1 & 0.2031 & 0.2015 \\
\hline 0.2 & 0.2098 & 0.2097 \\
\hline 0.3 & 0.2101 & 0.2122 \\
\hline 0.4 & 0.2119 & 0.2209 \\
\hline 0.5 & 0.2141 & 0.2216 \\
\hline 0.6 & 0.2197 & 0.2079 \\
\hline 0.7 & 0.2198 & 0.2267 \\
\hline 0.8 & 0.2193 & 0.2273 \\
\hline 0.9 & 0.2112 & 0.2367 \\
\hline
\end{tabular}

The general formula for computing the edge weights for the definition of the senses in [38] is coef $* \operatorname{minMax}\left(0, \alpha_{1}\right.$, ratio $)$, where the variable ratio is calculated as the number of times the term appears in the definition of the sense divided by the total number of non-noise words in the sense and $\alpha_{1}=0.6$. In our example, ratio = $1 / 2$ for both edges because we have only two non-noise words in the definition of the sense. The ratio parameter expresses the importance of the term in the definition of the sense. For example, if there are only two terms in the definition of the sense, then they are both very important. However, if there are 20 terms in the definition of the sense, then each individual term is less important. The minMax function makes the difference between the two cases less extreme. Using this function, the weight of the edge in the second case will be only roughly four times smaller than the weight of the edge in the first case. This is a common approach when processing text. The importance of a word in a document decreases as the size of the document increases, but the importance of the word decreases at a slower rate than the rate of the growth of the document. We use the minMax function every time we compare the number of occurrences of a term in a document compared to the total number of words in the document.

The minMax function returns a number that is in most cases between the first two arguments, where the magnitude of the number is determined by the third argument. Since the appearance of a term in the definition of a sense is not a reliable source of evidence about the relationship between the sense and the term, the value of the second argument is set to $\alpha_{1}<1$. The value for $\alpha_{1}$ is related to the probability that someone who is interested in a sense will also be interested in one of the terms in the definition of the sense.

Formally, the minMax function is defined as follows.

$\operatorname{minMax}(\min V a l u e$, maxValue, ratio)

$$
=\operatorname{minValue}+(\text { maxValue }- \text { minValue }) * \frac{-1}{\log _{2}(\text { ratio })} .
$$


Table 2. The value for the f-score for different values of $\alpha_{1}$ with and without using the $\operatorname{minMax}$ function.

\begin{tabular}{ccc}
\hline$\alpha_{1}$ & coef $* \min \operatorname{Max}\left(0, \alpha_{1}\right.$, ratio $)$ & coef $* \alpha_{1} *$ ratio \\
\hline 0.1 & 0.2031 & 0.2031 \\
0.2 & 0.2098 & 0.2023 \\
0.3 & 0.2101 & 0.2016 \\
0.4 & 0.2119 & 0.2100 \\
0.5 & 0.2141 & 0.2138 \\
0.6 & 0.2197 & 0.2143 \\
0.7 & $\mathbf{0 . 2 1 9 8}$ & $\mathbf{0 . 2 1 8 5}$ \\
0.8 & 0.2193 & 0.2183 \\
0.9 & 0.2112 & 0.2180 \\
\hline
\end{tabular}

Note that when ratio $=0.5$, the function returns maxValue. An unusual case is when the value of the variable ratio is bigger than 0.5 . For example, if ratio $=1$, then we have division by zero and the value for the function is undefined. We handle this case separately and assign value to the function equal to $1.2 *$ maxValue. This is an extraordinary case when there is a single non-noise word in the text description and we need to assign higher weight to the edge.

In our example, ratio $=\frac{1}{2}$ for both edges and therefore $\operatorname{minMax}\left(0, \alpha_{1}\right.$, ratio $)=$ $\alpha_{1}$ for both edges. An interesting question to ask is whether the minMax function makes a difference. As Table 2 shows, the answer is yes. The table shows the result of running our algorithm on the training set. The first column shows the results of using the minMax function and the second column shows the results without using the function. As the table suggests, using the minMax function does lead to bigger value for the f-score. We assume that this is also the case for the other edge weights formulas that use the minMax function in this paper and we do not run separate experiments to show the benefit of using the function for the rest of the formulas.

\subsection{Processing the example uses of the senses}

WordNet also includes example uses for each sense. For example, in WordNet the sentence "he put his coat over the back of the chair and sat down" is shown as an example use of the first sense of word "chair". Since the example use represents evidence that is weaker than the evidence from the definition of a sense, we will calculate the evidence probability as $\min \operatorname{Max}\left(0, \alpha_{2}\right.$, ratio $)$, where $\alpha_{2}<\alpha_{1}$. Here, the variable ratio is the number of times the term appears in the example use divided by the total number of non-noise words in the example use. The constant $\alpha_{2}$ is related to the probability that someone who is interested in a sense is also interested in one of the terms in the example use of the sense. The following edges are created from the first sense of the word "chair" and its example use. Note that the noise words have been omitted. 


$$
\begin{aligned}
& \text { a seat for one person } \Rightarrow \text { put; weight }=\operatorname{minMax}\left(0, \alpha_{2}, \frac{1}{5}\right)( \\
& \text { a seat for one person } \Rightarrow \text { coat; weight }=\operatorname{minMax}\left(0, \alpha_{2}, \frac{1}{5}\right) \\
& \text { a seat for one person } \Rightarrow \text { back; weight }=\operatorname{minMax}\left(0, \alpha_{2}, \frac{1}{5}\right)( \\
& \text { a seat for one person } \Rightarrow \text { sat; weight }=\operatorname{minMax}\left(0, \alpha_{2}, \frac{1}{5}\right)( \\
& \text { a seat for one person } \Rightarrow \text { down; weight }=\operatorname{minMax}\left(0, \alpha_{2}, \frac{1}{5}\right)
\end{aligned}
$$

The weight is the same for all edges because all words appear once in the example use. For all words, the value of ratio is equal to $\frac{1}{5}$. Note that we ignore the order of the words in the example use of a sense. In the algorithm from [38], the value for $\alpha_{2}$ is 0.2 . Next, let us examine how the value of $\alpha_{2}$ affects the value for the f-score. Table 3 shows how the results of changing only the value for $\alpha_{2}$ on our training set. The Table shows that this value indeed gives the optimal for the f-score and we will not change it.

Table 3. The value for the f-score for different values of $\alpha_{2}$.

\begin{tabular}{lc}
\hline$\alpha_{2}$ & f-score \\
\hline 0.1 & 0.2101 \\
0.2 & $\boldsymbol{0 . 2 1 9 7}$ \\
0.3 & 0.2086 \\
0.4 & 0.2032 \\
0.5 & 0.1989 \\
0.6 & 0.1948 \\
0.7 & 0.1941 \\
0.8 & 0.1927 \\
0.9 & 0.1922 \\
\hline
\end{tabular}

\subsection{Processing the backward edges}

We also create backward edges between a term and the sense that contain it in their definition. In [38], the weight of each edge is computed using the formula $\min \operatorname{Max}\left(0, \alpha_{3}\right.$, ratio $)$, where $\alpha_{3}=0.3$ and the variable ratio is the number of times the term appears in the definition of the sense divided by the total number of occurrences of the term in the definition of all senses. The constant $\alpha_{3}$ relates to the probability that someone who is interested in a term is also interested in one of the senses that have the term in their definition. As an example, if the word "position" occurs as part of the definition of only three senses and exactly once in each 
Table 4. The value for the f-score for different values of $\alpha_{3}$.

\begin{tabular}{cc}
\hline$\alpha_{3}$ & f-score \\
\hline 0.1 & 0.2122 \\
0.2 & 0.2131 \\
0.3 & $\mathbf{0 . 2 1 9 7}$ \\
0.4 & 0.2109 \\
0.5 & 0.2109 \\
0.6 & 0.2108 \\
0.7 & 0.2102 \\
0.8 & 0.2101 \\
0.9 & 0.2083 \\
\hline
\end{tabular}

definition, then we will add the following edge to the second sense of the word "chair".

$$
\text { position } \Rightarrow \text { the position of a professor; weight }=\operatorname{minMax}\left(0, \alpha_{3}, \frac{1}{3}\right) \text {. }
$$

We check to see what is the optimal value for the parameter $\alpha_{3}$. We ran our algorithm from [38] with different values for $\alpha_{3}$ on the training set and the results are shown in Table 4. As the table suggests, the optimal value is when $\alpha_{3}=0.3$, which is the value that is used in the algorithm from [40].

Similarly, the algorithm from [38] creates edges between terms and the senses that contain the terms in their example use. The weight of an edge in this case is computed as $\operatorname{minMax}\left(0, \alpha_{4}\right.$, ratio). Here, the ratio parameter is the number of times the term appears in the example use of the sense divided by the total number of occurrences in the example uses of all senses. The constant $\alpha_{4}$ relates to the probability that someone who is interested in a term is also interested in one of the senses that have the term in their example use. As an example, if the word "coat" occurs as part of the example use of only three senses and exactly once in each sense, then we will add the following edge to the first sense of the word "chair". Note that the example use of this sense is: "he put his coat over the back of the chair and sat down".

$$
\text { coat } \Rightarrow \text { a seat for one person; weight }=\operatorname{minMax}\left(0, \alpha_{4}, \frac{1}{3}\right) .
$$

In [38], the value for $\alpha_{4}$ is set to 0.1 . Table 5 shows that this is the optimal value for our training set.

\subsection{Populating the frequencies of the senses}

So far, we have shown how to extract information from textual sources, such as the text for the definition and example use of a sense. We will next show how structured knowledge, such as the hyponym (a.k.a. kind-of) relationship between senses, can be represented in the similarity graph. Most existing approaches [29] explore these 
Table 5. The value for the f-score for different values of $\alpha_{4}$.

\begin{tabular}{cc}
\hline$\alpha_{4}$ & f-score \\
\hline 0.1 & $\boldsymbol{O . 2 1 9 7}$ \\
0.2 & 0.2131 \\
0.3 & 0.2169 \\
0.4 & 0.2177 \\
0.5 & 0.2194 \\
0.6 & 0.2152 \\
0.7 & 0.2106 \\
0.8 & 0.2104 \\
0.9 & 0.2088 \\
\hline
\end{tabular}

relationships by evaluating the information content of different terms. Here, we adjust this approach and focus on the frequency of use of each word in the English language as described in the University of Oxford's British National Corpus. The description of this corpus, as presented in [3], is: "The British National Corpus is a 100 million word collection of samples of written and spoken language from a wide range of sources, designed to represent a wide cross-section of British English, both spoken and written, from the late twentieth century."

Let $s$ be a sense. Let $\left\{w f_{i}\right\}_{i=1}^{n}$ be the word forms for that sense. We will use $B N C(w f)$ to denote the frequency of the word form in the British National Corpus. Let $p_{s}(w f)$ be the frequency of use of the sense $s$ of the word form $w f$, as specified in WordNet, divided by the sum of the frequencies of use of all senses of $w f$ (also as defined in WordNet). Then we define the size of $s$ to be equal to $|s|=\sum_{i=1}^{n}\left(B N C\left(w f_{i}\right) * p_{s}\left(w f_{i}\right)\right)$.

The ebove formula approximates the size of a sense by looking at all the word forms that represent the sense and figuring out how much each word form contributes to the sense. The size of a sense approximates its popularity. For example, according to WordNet, the word "president" has six different senses with frequencies: $14,5,5,3,3$, and 1 . Let us refer to the fourth sense: "The officer who presides at the meetings ..." as $s$. According to above definition, $p_{s}$ (president) $=3 / 31=0.096$ because the frequency of $s$ is 3 and the sum of all the frequencies is 31 . Since the British National Corpus shows the frequency of the word "president" as 9781 , the contribution of the word "president" to the size of the sense $s$ is equal to $|s|=B N C($ president $) * p_{s}($ president $)=9781 * 0.096=938.98$. Other terms that represent the sense $s$, such as "chairman", will also contribute to the size of the sense.

\subsection{Processing structured knowledge about nouns}

WordNet defines the hyponym (a.k.a. kind-of) relationship between senses that represent nouns. For example, the most popular sense of the word "dog" is a hyponym of the most popular sense of the word "canine". Consider the first sense of the word "chair": "a seat for one person". WordNet defines 15 hyponyms for this 
sense, including senses for the words "armchair" and "wheelchair". We will add edges that show the conditional probability between this first sense of the word "chair" and each of the hyponyms. Let the probability that someone who is interested in a sense is also interested in one of the sub-senses be equal to $\alpha_{5}$. In order to determine the weight of each edge, we need to compute the size of each sense. In the British National Corpus, the frequency of "armchair" is 657 and the frequency of "wheelchair" is 551. Since both senses are associated with a single term, we do not need to consider the frequency of use of each sense. If "armchair" and "wheelchair" were the only hyponyms of the sense "a seat for one person", then we need to add the following edges.

a seat for one person $\Rightarrow$ chair with support on each side for arms; weight $=\alpha_{5} * 657 / 1208$

a seat for one person $\Rightarrow$ a moveable chair mounted on large wheels; weight $=\alpha_{5} * 551 / 1208$

In general, the weight of an edge in [38] is computed as $\alpha_{5}$ multiplied by the size of the sense and divided by the sum of the sizes of all the hyponym senses of the initial sense. The idea is that the the weight of an edge to a "bigger" sense will be bigger because it is more likely that a bigger sense is relevant. Note that here we do not apply the minMax function. The reason is that the function is only relevant when computing the ratio of the number of occurrences of a term in text relative to the size of the text. In [38], the value of $\alpha_{5}=0.9$ was used. However, as the table Table 6 suggests, the value of $\alpha_{5}=0.2$ is optimal for our training set.

We will also create edges for the hypernym relationship (the inverse of the hyponym relationship). For example, the first sense of the word "canine" is a hypernym of the first sense of the word "dog". The weight for each edge is the same and equal to $\alpha_{6}$. This represents the probability that someone who is interested in a sense will be also interested in the hypernyms of the sense. For example, if a user is interested in the sense "wheelchair", then they may be also interested in the first

Table 6. The value for the f-score for different values of $\alpha_{5}$.

\begin{tabular}{lc}
\hline$\alpha_{5}$ & f-score \\
\hline 0.1 & 0.2196 \\
0.2 & $\mathbf{0 . 2 2 0 0}$ \\
0.3 & 0.2197 \\
0.4 & 0.2197 \\
0.5 & 0.2196 \\
0.6 & 0.2197 \\
0.7 & 0.2197 \\
0.8 & 0.2197 \\
0.9 & 0.2197 \\
\hline
\end{tabular}


Table 7. The value for the f-score for different values of $\alpha_{6}$.

\begin{tabular}{cc}
\hline$\alpha_{6}$ & f-score \\
\hline 0.1 & 0.2258 \\
0.2 & $\mathbf{0 . 2 2 5 8}$ \\
0.3 & 0.2197 \\
0.4 & 0.2198 \\
0.5 & 0.2198 \\
0.6 & 0.2198 \\
0.7 & 0.2204 \\
0.8 & 0.2204 \\
0.9 & 0.2204 \\
\hline
\end{tabular}

sense of the word chair. However, this probability is not a function of the different hypernyms of the sense. Here is the example edge that will be built.

$$
\begin{aligned}
\text { chair with support on each side for arms } \Rightarrow & \text { a seat for one person; } \\
& \text { weight }=\alpha_{6}
\end{aligned}
$$

Table 7 shows how the value of $\alpha_{6}$ affects the f-score for our testing data. The value of $\alpha_{6}=0.3$ was used in [38], which is not the optimal value.

We next consider the meronym (a.k.a. part-of) relationship between nouns. Note that we do not make a distinction between the three types of meronyms (part, member, and substance) and process them identically. For example, WordNet contains information that the sense of the word "back": "a support that you can lean against ..." and the sense of the word "leg": "one of the supports for a piece of furniture" are both meronyms of the first sense of the word "chair". In other words, back and legs are building parts of a chair. Part of this information can be represented using the following edges.

a seat for one person $\Rightarrow$ a support that you can lean against; weight $=\alpha_{7} / 2$

a seat for one person $\Rightarrow$ one of the supports for a piece of furniture;

$$
\text { weight }=\alpha_{7} / 2
$$

In general, we compute the weight on an edge as $\alpha_{7} / n$, where $n$ is the number of meronyms of the sense. The reasoning behind the formula is that the more meronyms a sense has, the less likely it is that we are interested in a specific meronym. Table 8 shows how the value of $\alpha_{7}$ affects the f-score for our testing data. The value of $\alpha_{7}=0.6$ was used in [38], which is not the optimal value. Note that the meronym relationship is very rear in WordNet and therefore tuning the $\alpha_{7}$ parameter does not significantly affect the resulting graph.

We also represent the holonym (the reverse of the meronym) relationship between nouns. For example, the main sense of the word "building" is a holonym of the main sense of the word "window". We set the weight of each each to a constant: $\alpha_{8}$ and therefore create the following edges for our example. 
Table 8. The value for the f-score for different values of $\alpha_{7}$.

\begin{tabular}{ll}
\hline 0.1 & 0.2189 \\
0.2 & $\mathbf{0 . 2 1 9 8}$ \\
0.3 & 0.2197 \\
0.4 & 0.2197 \\
0.5 & 0.2197 \\
0.6 & 0.2197 \\
0.7 & 0.2197 \\
0.8 & 0.2197 \\
0.9 & 0.2197 \\
\hline
\end{tabular}

$$
\begin{aligned}
\text { a support that you can lean against } \Rightarrow & \text { a seat for one person; } \\
& \text { weight }=\alpha_{8} \\
\text { one of the supports for a piece of furniture } \Rightarrow & \text { a seat for one person; } \\
& \text { weight }=\alpha_{8}
\end{aligned}
$$

Table 9 shows how the value of $\alpha_{8}$ affects the f-score for our testing data. The value of $\alpha_{8}=0.1$ was used in [38] and it is the optimal value. Note that the holonym relationship is very rarely used in WordNet and therefore the value of $\alpha_{8}$ does not affect the value of the f-score.

\begin{tabular}{lc}
$\begin{array}{l}\text { Table 9. The value for the f-score } \\
\text { for different values of } \alpha_{8} \text {. }\end{array}$ \\
\hline 0.1 & $\mathbf{0 . 2 1 9 7}$ \\
0.2 & 0.2197 \\
0.3 & 0.2197 \\
0.4 & 0.2197 \\
0.5 & 0.2197 \\
0.6 & 0.2197 \\
0.7 & 0.2197 \\
0.8 & 0.2197 \\
0.9 & 0.2197 \\
\hline
\end{tabular}

\subsection{Processing structured knowledge about verbs}

We will first represent the troponym (a.k.a. doing in some manner) relationship for verbs. For example, to lisp is a troponym of to talk. Suppose that the main sense of the verb "talk" has only three troponyms: "lisp", "orate", and "converse". If the sizes of the main senses of the three verbs are 18, 1, and 95 (as determined by the formula for the size of a sense in Sec. 3.5), respectively, then we will create the following edges.

$$
\begin{array}{r}
\text { an exchange of ideas via conversation } \Rightarrow \text { talk with a lisp; } \\
\qquad \text { weight }=\alpha_{9} * \frac{18}{114}
\end{array}
$$


an exchange of ideas via conversation $\Rightarrow$ talk pompously;

$$
\text { weight }=\alpha_{9} * \frac{1}{114}
$$

an exchange of ideas via conversation $\Rightarrow$ carry on a conversation;

$$
\text { weight }=\alpha_{9} * \frac{95}{114}
$$

The edges are from the first sense of the word "talk": "an exchange of ideas via conversation". The destination nodes are for the first senses of "lisp", "orate" and "converse", respectively. For example, the first edge expresses the conditional probability between the main senses for "talk" (an exchange of ideas via conversation) and the main sense for "lisp" (talk with a lisp). The constant $\alpha_{9}$ represents the probability that someone who is interested in a verb is also interested in one of its troponyms. The weight of each sense is computed as $\alpha_{9}$ multiplied by the size of the sense and divided by the sum of the sizes of all the troponym senses. Table 10 shows that the value of $\alpha_{9}=0.9$ that was picked in [38] does not produce the highest value for the f-score for our training data.

We will also add edges for the reverse relationship with constant weight of $\alpha_{10}$ for all edges. For example, we will add the following edge.

$$
\text { talk with a lisp } \Rightarrow \text { an exchange of ideas via conversation; weight }=\alpha_{10}
$$

This means that if someone is interested in one of the troponyms, then there is a $\alpha_{10}$ probability that they are also interested in the original verb. In [38], the value of $\alpha_{10}=0.3$ was used. However, Table 11 shows that the optimal value for the training set is $\alpha_{10}=0.7$.

The hyponym and hypernym relationships are defined not only for nouns, but also for verbs. The two relationships are the reverse of each other. In other words, if $\mathrm{X}$ is a hyponym of $\mathrm{Y}$, then $\mathrm{Y}$ is a hypernym of $\mathrm{X}$. The hypernym relationship for verbs corresponds to the "one way to" relationship. For example, the verb "perceive" is the hypernym of the verb "listen" because one way of perceiving something is by listening. As expected, the verb "listen" is a hyponym of the verb "perceive". The first sense of the word "perceive" is "to become aware of through the senses". Suppose

Table 10. The value for the f-score for different values of $\alpha_{9}$.

\begin{tabular}{lc}
\hline 0.1 & 0.2244 \\
0.2 & 0.2244 \\
0.3 & 0.2244 \\
0.4 & 0.2244 \\
0.5 & 0.2244 \\
0.6 & $\mathbf{0 . 2 2 4 5}$ \\
0.7 & 0.2197 \\
0.8 & 0.2197 \\
0.9 & 0.2197 \\
\hline
\end{tabular}


Table 11. The value for the f-score for different values of $\alpha_{10}$.

\begin{tabular}{ll}
\hline 0.1 & 0.2195 \\
0.2 & 0.2197 \\
0.3 & 0.2197 \\
0.4 & 0.2197 \\
0.5 & 0.2202 \\
0.6 & 0.2186 \\
0.7 & $\mathbf{0 . 2 2 4 0}$ \\
0.8 & 0.2214 \\
0.9 & 0.2197 \\
\hline
\end{tabular}

that the first senses of the verbs "listen" and "see" are the only hypernyms of the verb "perceive".

We will assume that the probability that someone who is interested in a verb sense is also interested in one of the hyponym senses is equal to $\alpha_{11}$. In order to determine the weights of the edges, we need to compute the size of each sense. In the British National Corpus, the frequency of "listen" is 1241 and the frequency of "see" is 3624 . Since both senses are associated with a single word form, we do not need to consider the frequency of use of each sense. If "perceive" and "see" were the only hyponyms of the sense "to become aware of thought and senses", then we will create the following edges.

to become aware of thought and senses $\Rightarrow$ pay attention to sound;

$$
\text { weight }=\alpha_{11} * \frac{1241}{4865}
$$

to become aware of thought and senses $\Rightarrow$ percieve by sight;

$$
\text { weight }=\alpha_{11} * \frac{3624}{4865}
$$

In general, the weight of each edge is computed as the size of the sense divided by the sum of sizes of all hyponym senses. The idea behind the formula is that the the weight of an edge to a "bigger" senses will be bigger because it is more likely that such bigger senses are relevant. Table 12 shows how $\alpha_{11}$ affects the value of the f-score for the training data. In [38], the value of $\alpha_{11}=0.9$ was used, which turns out to be optimal for the training data.

We will use edge weights of $\alpha_{12}$ for the hypernym (the reverse of the hyponym) relationship. For example, the main sense of the verb "perceive" is a hypernym of the main senses of the verbs "listen" and "see". This information can be expressed using the following edges.

pay attention to sound $\Rightarrow$ to become aware of thought and senses; weight $=\alpha_{12}$ percieve by sight $\Rightarrow$ to become aware of thought and senses; weight $=\alpha_{12}$

The coefficient $\alpha_{12}$ represents the probability that someone who is interested in a sense will also be interested in the hypernyms of the sense. For example, if a user is 
Table 12. The value for the f-score for different values of $\alpha_{11}$.

\begin{tabular}{lc}
\hline 0.1 & 0.2124 \\
0.2 & 0.2124 \\
0.3 & 0.2124 \\
0.4 & 0.2133 \\
0.5 & 0.2171 \\
0.6 & 0.2171 \\
0.7 & 0.2185 \\
0.8 & $\mathbf{0 . 2 1 9 7}$ \\
0.9 & 0.2197 \\
\hline
\end{tabular}

interested in the sense "see", then they may be also interested in the first sense of the word perceive. However, this probability is not a function of the different hypernyms of the sense.

Table 13 shows how $\alpha_{12}$ affects the value of the f-score for the training data. In [38], the value of $\alpha_{12}=0.3$ was used, which turns out to be close to the optimal value of $\alpha_{12}=0.1$.

\begin{tabular}{ll}
$\begin{array}{l}\text { Table 13. The value for the f-score } \\
\text { for different values of } \alpha_{12} \text {. }\end{array}$ \\
\hline 0.1 & $\mathbf{0 . 2 2 4 5}$ \\
0.2 & 0.2197 \\
0.3 & 0.2197 \\
0.4 & 0.2198 \\
0.5 & 0.2198 \\
0.6 & 0.2198 \\
0.7 & 0.2198 \\
0.8 & 0.2198 \\
0.9 & 0.2198 \\
\hline
\end{tabular}

\subsection{Processing structured knowledge about adjectives}

WordNet defines two relationships for adjectives: related to and similar to. For example, the first sense of the adjective "slow" has definition: "not moving quickly", while the first sense of the adjective "fast" has the definition: "acting or moving or capable of acting or moving quickly". WordNet specifies that the two senses are related to each other. We will represent this relationship using the following edges.

$$
\begin{aligned}
& \text { not moving quickly } \Rightarrow \text { acting or moving quickly; weight }=\alpha_{13} \\
& \text { acting or moving quickly } \Rightarrow \text { not moving quickly; weight }=\alpha_{13}
\end{aligned}
$$

This represents that there is a $\alpha_{13}$ probability that someone who is interested in an adjective is also interested in a "related to" adjective. In [38], $\alpha=0.6$. Table 14 shows that this is close to the the optimal value for our training data. 
Table 14. The value for the f-score for different values of $\alpha_{13}$.

\begin{tabular}{ll}
\hline 0.1 & 0.2197 \\
0.2 & 0.2197 \\
0.3 & 0.2197 \\
0.4 & 0.2198 \\
0.5 & 0.2197 \\
0.6 & 0.2197 \\
0.7 & $\mathbf{0 . 2 1 9 8}$ \\
0.8 & 0.2198 \\
0.9 & 0.2198 \\
\hline
\end{tabular}

Table 15. The value for the f-score for different values of $\alpha_{14}$.

\begin{tabular}{ll}
\hline 0.1 & 0.2199 \\
0.2 & 0.2199 \\
0.3 & 0.2199 \\
0.4 & $\mathbf{0 . 2 1 9 9}$ \\
0.5 & 0.2197 \\
0.6 & 0.2198 \\
0.7 & 0.2197 \\
0.8 & 0.2197 \\
0.9 & 0.2197 \\
\hline
\end{tabular}

WordNet also defines the similar to relationship between adjectives. We create edges with weights of $\alpha_{14}$ for this relationship, where $\alpha_{14}=0.8$ in [38]. The number corresponds to the probability that someone who is interested in an adjective is also interested in a "similar to" adjective. For example, WordNet contains the information that the sense for the word "frequent": "coming at short intervals" and the sense for the word "prevailing": "most frequent or common" are similar to each other. We will therefore create the following edges.

coming at short intervals $\Rightarrow$ most frequent or common; weight $=\alpha_{14}$ most frequent or common $\Rightarrow$ coming at short intervals; weight $=\alpha_{14}$

Note that both the "similar to" and "related to" relationships are symmetric and therefore the weight of an edge and its reverse is the same. Table 15 shows how the value of $\alpha_{14}$ affects the f-score on our training data. Note that both the "similar to" and "related to" relationships are very rear in WordNet and therefore the edges for them do not significantly influence the value for the f-score.

\section{Measuring the Semantic Similarity Between Terms}

The similarity graph is used to estimate the conditional probability that a user is interested in the term that is described by the label of a node given that they are also 
interested in the label of an adjacent node in the graph. Note that in some case the weight of an edge can become more than one, in which case we restrict it to one in order to be a probability. We compute the directional similarity between two nodes using the following formula.

$$
\begin{gathered}
A \rightarrow{ }_{s} C=\sum_{P t \text { is a cycleless path fron }(\text { node } A \text { to node } C} P_{P t}(C \mid A) \\
P_{P t}(C \mid A)=\prod_{\left(n_{1}, n_{2}\right) \text { is an edge ip the path } P t} P\left(n_{2} \mid n_{1}\right) .
\end{gathered}
$$

The function $P\left(n_{2} \mid n_{1}\right)$ refers to the weight of the edge from the node $n_{1}$ to the node $n_{2}$. Informally, we compute the directional similarity between two nodes as the sum of the weights of all the paths between the two nodes, where we eliminate cycles from the paths. Each path provides evidence about the similarity between the terms that are represented by the two nodes. We compute the weight of a path between two nodes as the product of the weights of the edges along the path, which follows the Markov chain model. Since the weight of an edge along the path is almost always smaller than one (i.e., equal to one only in rear circumstances), the value of the conditional probability will decrease as the length of the path increases. This is a desirable behavior because a longer path provides less evidence about the similarity of the two end nodes. For alternative ways of computing the directional similarity between two nodes, see [39]. Note that there can be multiple interweaving paths between two nodes. The above algorithms finds disjoint paths (i.e., paths with no edges in common) and there are multiple ways to do so. As expected, the decision of which paths to pick affects the clustering algorithm.

Next, we present two functions for measuring the semantic similarity between two nodes. The linear function for computing the semantic similarity between two nodes is shown in Eq. (5).

$$
\left|w_{1}, w_{2}\right|_{\text {lin }}=\min \left(\alpha, \frac{w_{1} \rightarrow{ }_{s} w_{2}+w_{2} \rightarrow{ }_{s} w_{1}}{2}\right) * \frac{1}{\alpha}
$$

The minimum function is used in order to cap the value of the similarity function at one. The coefficient $\alpha$ amplifies the available evidence $(\alpha \leq 1)$. Note that when $\alpha$ is equal to one, then the function simply takes the average of the two numbers and caps the result at 1 .

The second similarity function is inverse logarithmic, that is, it amplifies the smaller values. It is shown in Eq. (6). The norm function simply multiplies the result by a constant (i.e., $\left.-\log _{2}(\alpha)\right)$ in order to move the result value in the range $[0,1]$.

$$
\left.\left|w_{1}, w_{2}\right|_{\text {log }}=\text { norm } \frac{-1}{\log _{2}\left(\min \left(\alpha, \frac{w_{1} \rightarrow s}{w_{2}+w_{2} \rightarrow w_{1}}\right)\right)}\right) \cdot(
$$

The paper [39] suggests that the two similarity metrics produce best results when $\alpha$ is around 0.1 and this is the value that is used in the experimental section. Note 
that since both metrics are monotonic, the value for $\alpha$ or the choice of similarity function does not affect the result of the clustering algorithm.

\section{Measuring the Semantic Similarity Between Documents}

In the previous section, we described how to measure the semantic similarity between two nodes of the graph. In this section, we describe how to measure the semantic similarity between any two text documents. The idea is to create a node for each document and then connect the nodes to the graph. The semantic similarity between two documents will then be measured by computing the distance between the two nodes using the linear or logarithmic metric from the previous section.

In order to demonstrate our approach, consider a fictitious document that contains a total of 10 non-noise words in its title and a total of 100 non-noise words in its body. Among these non-noise words, suppose that the word "sneaker" appears once in the title and the word "shirt" is present four times in the body. We will represent this information by creating the following edges.

$$
\begin{aligned}
& \text { document } \Rightarrow \text { sneakers } ; \text { weight }=\text { computeMinMax }\left(0, \alpha_{15}, 1 / 10\right) \\
& \text { document } \Rightarrow \text { shirt } ; \text { weight }=\text { computeMinMax }\left(0, \alpha_{16}, 4 / 100\right)
\end{aligned}
$$

The weights of the edges are computed similar to the way the weights of the edged between a sense and the words in its definition were computed. The number $\alpha_{15}$ is used to represent the probability that someone who is interested in a document also wants information about one of the terms that appears in its title. The number $\alpha_{16}$ represents the probability that someone who is interested in a document is also interested in one of the terms that appear in its body. In [38], we set $\alpha_{15}$ to 0.6 and $\alpha_{16}$ to 0.3 because of our belief that terms in the title of a document are twice as important.

Table 16 shows that the value of $\alpha_{15}=0.9$ is optimal for our test set. In other words, the tuning shows that someone who is interested in a document is almost always interested in one of the words in its title. Table 17 shows that the value of $\alpha_{16}=0.9$ is optimal for our test set. Again, the tuning shows that someone who is interested in a document is almost always interested in one of the words in the text.

Table 16. The value for the f-score

\begin{tabular}{ll} 
for different values of $\alpha_{15}$. \\
\hline 0.1 & 0.1913 \\
0.2 & 0.1896 \\
0.3 & 0.2037 \\
0.4 & 0.1921 \\
0.5 & 0.2054 \\
0.6 & 0.2197 \\
0.7 & 0.2299 \\
0.8 & 0.2360 \\
0.9 & $\mathbf{0 . 2 7 2 7}$ \\
\hline
\end{tabular}


Table 17. The value for the f-score for different values of $\alpha_{16}$.

\begin{tabular}{ll}
\hline 0.1 & 0.2466 \\
0.2 & 0.2491 \\
0.3 & 0.2197 \\
0.4 & 0.2071 \\
0.5 & 0.2300 \\
0.6 & 0.2743 \\
0.7 & 0.2607 \\
0.8 & 0.2546 \\
0.9 & $\mathbf{0 . 2 7 5 1}$ \\
\hline
\end{tabular}

Next, consider the backward edge between the word "sneaker" and the document. Suppose that the word appears a total of 10 times in the title of documents. Then the weight of the edge between the word "sneaker" and a document that contains the word in the its title will be equal to $\alpha_{17} \cdot \frac{1}{10}$. This is the same formula that is used for computing the weights of the backward edges between a word form from WordNet and the definition of the sense in which it appears, but the value of the coefficient is different. Similarly, if the word "shirt" appears a total of 20 times in the body of documents and four times in the body of our document, then we will draw a backward edge with weight $\alpha_{18} \cdot \frac{4}{20}$ between the word and the document. In [38], the value of $\alpha_{17}$ is 0.3 and the value of $\alpha_{18}$ is 0.15 . Table 18 shows that the value of $\alpha_{17}=0.3$ is not optimal. On our training set, the optimal value is when $\alpha_{17}=0.7$. Similarly, Table 19 shows that the optimal value for $\alpha_{18}$ over our training set is when $\alpha_{18}=0.4$. Our assumption that the words in the title of a document are roughly twice as important as words in the text of the document turns out to be correct in this case.

Note that we do not pay special attention to the order of the words. The reason is that there is no empirical evidence that the first words in the title or body of a document are more important.

The word "sneaker" has two different senses. Our algorithm does not try to identify which of these senses the document refers to. Instead, there will be paths in the graph to both senses. We take this approach because it can be possible that

Table 18. The value for the f-score for different values of $\alpha_{17}$.

\begin{tabular}{ll}
\hline 0.1 & 0.1767 \\
0.2 & 0.2191 \\
0.3 & 0.2197 \\
0.4 & 0.1968 \\
0.5 & 0.2046 \\
0.6 & 0.2338 \\
0.7 & $\mathbf{0 . 2 4 0 7}$ \\
0.8 & 0.1844 \\
0.9 & 0.1999 \\
\hline
\end{tabular}


Table 19. The value for the f-score

for different values of $\alpha_{18}$.

\begin{tabular}{ll}
\hline 0.1 & 0.1897 \\
0.2 & 0.2260 \\
0.3 & 0.3157 \\
0.4 & $\mathbf{0 . 3 3 2 7}$ \\
0.5 & 0.3091 \\
0.6 & 0.3047 \\
0.7 & 0.2771 \\
0.8 & 0.2532 \\
0.9 & 0.2455 \\
\hline
\end{tabular}

different occurrences of the word in the document refer to distinct senses. The strength of the relationship to a particular sense will be computed based on additional evidence. For example, if the document also contains the word "shoe", then there will be stronger connection between the document and the main sense of the word "sneaker".

Second, note that the distance between two documents is not calculated in isolation. In particular, the other documents in the corpus are also taken into account when calculating the backward edges. In other words, we calculate how similar two documents are relative to the rest of the documents in the corpus. Once the similarity graph is extended with the documents, the distance between two documents can be calculated using the linear or logarithmic metrics that were described in the previous section.

\section{Clustering the Documents}

In this section we describe how a set of documents can be clustered using the $k$-means clustering algorithm. The algorithm relies on a way for computing the distance between two documents. We present two variations: using keywords matching and using the similarity graph. In the next section we show how the results of the two algorithms compare to human judgment and the effect of tuning the parameters of the second algorithm.

A common approach for computing the similarity distance between two documents is to represent them as vectors and then compute the cosine of the angle between the two vectors as the normalized dot product of the vectors. For example, suppose that "dog", "cat" and "shirt" are the only words that are used. Then for every document we can denote the number of times each word occurs. For example, a document that contains the word "dog" twice, the word "cat" three times and does not contain the word "shirt" can be represented as the document vector $[2,3,0]$. Alternatively, a document that contains the word "cat" twice and the word "shirt" four times can be represented as $[0,2,4]$. The dot product of the two vectors is $[2,3,0] \cdot[0,2,4]=6$. Next, we need to divide the result by the product of the sizes of the two documents. Therefore, the angle between the documents in radians will be 
$\frac{6}{\sqrt{\left(2^{2}+3^{2}\right)} * \sqrt{2^{2}+4^{2}}} \approx 0.37$. Unfortunately, this approach does not take into account that the words "cat' and "dog" are semantically similar and will calculate the similarity distance between a document about cats and one about dogs as zero if the two documents do not share words in common. In general, the cosine similarity between two documents is computed using the formula in Eq. (7). Alternatively, we can use the linear or logarithmic metric from the previous section to compute the semantic distance between two documents.

$$
\left|d_{1}, d_{2}\right|_{\text {cosine }}=\frac{\vec{d}_{1} \cdot \vec{d}_{2}}{\left|\vec{d}_{1}\right| *\left|\vec{d}_{2}\right|} .
$$

The $k$-means clustering algorithm starts with a constant $k$. This is the number of clusters that will be produced. Initially, a centroid (i.e., a document) is randomly chosen for every cluster. Next, each document is assigned to the group that contains the closest centroid. After that, the centroid (i.e., mean) is found for each cluster and then the documents are clustered again around each centroid. The algorithm continues until applying the last step does not change the clustering. If the documents are represented as vectors, as we showed earlier in this section, then computing the mean of a set of documents amounts to adding the document vectors and dividing by the number of documents. For example, the mean of our two document vectors from the beginning of this section is mean $([2,3,0],[0,2,4])=\frac{[2,5,4]}{2}=[1,2.5,2]$. Note that the mean function is independent of the document similarity metric that is used.

\section{Experimental Results}

All our code was implemented in Java. We first created the similarity graph using WordNet and it took about $10 \mathrm{~min}$ to create the graph on a standard laptop with Intel i5 CPU. We used the Java API for WordNet Searching (JAWS) to connect to WordNet. The interface was developed by Brett Spell [35]. We stored the graph as several Java hash tables, where the size of the file is $89 \mathrm{MB}$.

We next read 9,362 documents from the Reuters-21578 benchmark and added them to similarity graph. The benchmark contains 21,578 documents that are stored in 22 text files. Out of those documents, 11,362 documents are classified in one of 82 categories using human judgment. Out of those 11,362 documents, 2,000 were used as a training set to adjust the parameters of our algorithm. All experimental results in this section are done on the remaining 9, 362 documents. For every document, we stored its title, its text, the category it belongs to, and a document vector. The later contains the non-noise words in the documents and their frequency. Since the words in the title are more important, we counted these words twice. We stored the information in Java hash tables, where the size of the file is $22 \mathrm{MB}$. It took about two minutes to parse the text files.

We next added the documents to the similarity graph. We followed the algorithm from Sec. 5. The size of the graph increased to $121 \mathrm{MB}$. For document nodes, we 
stored the title of the document in the label of the node. We also stored a hash table that keeps the mapping between the document nodes in the graph and the documents in the document file that was described in the previous paragraph. It took about five minutes to add the documents to the graph.

We next clustered the documents using the $k$-means clustering algorithm. We chose the value $k=82$ because this is the number of categories as determined by the human judgment. The first 82 documents were put in 82 distinct clusters. At this point, the lonely document in each category was designed as the centroid. We next processed the rest of the documents. Every document was compared to the 82 centroids and assigned to the cluster with the closest centroid. Next, a new centroid was chosen for each cluster. This was done by adding the document vectors in each cluster and dividing the result by the number of vectors (i.e., finding the mean in each cluster). Next, the documents were reclustered around the new centroids and the process was repeated until it converged (i.e., applying the algorithm did not change the clusters).

The $k$-means clustering algorithm is based on two document functions: finding the distance between two documents and computing the average of several documents. The later function is implemented by simply adding the document vectors and dividing the result by the number of vectors. However, we have three choices for the distance metric: the cosine, linear, and logarithmic. When we applied the cosine similarity metric, the $k$-means clustering program terminated in about three hours. Note that since the linear and logarithmic function are both monotonic, we got exactly the same results using either function.

Table 20 shows the precision, recall, f-score, entropy, and purity when using the three different algorithms. Table 21 summarizes the differences between the algorithm from [38] and the tuned-up version (i.e., columns 2 and 3 in the table.) Note that the first criteria is whether we consider the first words in the definition of the sense to be more important, that is whether we multiply the weight of the edges by the coef variable that decreases with each consecutive word in the definition of a sense. The fine-tuned version significantly changes the values for some of the parameters, where the values in the original algorithm [38] were estimated. However, as Table 20 shows, fine-tuning the parameters leads to significantly better results.

We will next show the formulas for computing the precision, recall, f-score, entropy, and purity. Let TP be the number of true positives, that is, the number of documents that were classified in the same category by both the program and human

Table 20. Summary of results on the experimental data set.

\begin{tabular}{lccc}
\hline & Cosine metric & Similarity graph & Fine-tuned similarity graph \\
\hline precision & 0.53 & 0.58 & 0.66 \\
recall & 0.06 & 0.08 & 0.10 \\
f-score & 0.10 & 0.14 & 0.17 \\
entropy & 1.75 & 1.76 & 1.72 \\
purity & 0.66 & 0.67 & 0.69 \\
\hline
\end{tabular}


Table 21. Differences between the original and the fine-tuned similarity graph algorithm.

\begin{tabular}{lcc}
\hline & Algorithm from [38] & Fine-tuned version \\
\hline Consider word ordering & yes & no \\
Use minMax & yes & yes \\
$\alpha_{1}$ & 0.6 & 0.7 \\
$\alpha_{2}$ & 0.2 & 0.2 \\
$\alpha_{3}$ & 0.3 & 0.3 \\
$\alpha_{4}$ & 0.1 & 0.1 \\
$\alpha_{5}$ & 0.9 & 0.2 \\
$\alpha_{6}$ & 0.3 & 0.2 \\
$\alpha_{7}$ & 0.6 & 0.2 \\
$\alpha_{8}$ & 0.1 & 0.1 \\
$\alpha_{9}$ & 0.9 & 0.6 \\
$\alpha_{10}$ & 0.3 & 0.7 \\
$\alpha_{11}$ & 0.8 & 0.8 \\
$\alpha_{12}$ & 0.3 & 0.1 \\
$\alpha_{13}$ & 0.6 & 0.7 \\
$\alpha_{14}$ & 0.8 & 0.4 \\
$\alpha_{15}$ & 0.6 & 0.9 \\
$\alpha_{16}$ & 0.3 & 0.9 \\
$\alpha_{17}$ & 0.3 & 0.7 \\
$\alpha_{18}$ & 0.15 & 0.4 \\
\hline
\end{tabular}

judgment. Let FP be the number of false positives, that is, the number of documents that were classified in the same category by the program, but were classified in different categories by human judgment. Lastly, let FN be the number of false negatives, that is, the number of documents that were classified in the same category by human judgment but were classified in different categories by the program. The formulas for computing the precision, recall, and f-score are shown below.

$$
\begin{gathered}
P=\frac{T P}{T P+F P} \\
R=\frac{T P}{T P+F N} \\
F_{\beta}=\frac{\left(\beta^{2}+1\right) \cdot P \cdot R}{\beta^{2} \cdot P+R}
\end{gathered}
$$

In the above formulas, $P$ is used to denote the precision and $R$ is used to denote the recall. We used the value $\beta=1$ in the experimental results, which is a popular parameter for the f-score.

Entropy can be used to measure the diversity of the result, where lower entropy means that the documents in the computer-generated cluster are more similar, that is more of them belong to the same human-determined cluster. An entropy of zero means exact match. For each cluster $D_{i}$ that is generated by our algorithm, we can compute the entropy as entropy $\left(D_{i}\right)=-\sum_{j=1}^{k} \operatorname{Pr}_{i, j} \cdot \log _{2}\left(\operatorname{Pr}_{i, j}\right)$, where $\operatorname{Pr}_{i, j}$ is the proportion (relative to the total size of cluster $D_{i}$ ) of data from cluster $D_{j}$ (as 
determined by the human judgment) that ended up in cluster $D_{i}$. The total entropy can be computed as the weighted average of the entropies of all clusters, or more precisely using the formula: $\sum_{i=1}^{k} \frac{\left|D_{i}\right|}{|D|} \cdot \operatorname{entop} y\left(D_{i}\right)$. Note that we have used $\left|D_{i}\right|$ to denote the number of documents in cluster $D_{i}$ and $|D|$ to denote the total number of documents.

Lastly, purity measure the extends that a computer-generated cluster contains pure data, that is documents from the same human-defined cluster. A purity of one means exact match. Formality, for a computer-generated cluster of documents $D_{i}$, we define purity $\left(D_{i}\right)=\max _{j=1}^{k}\left(\operatorname{Pr}_{i, j}\right)$. The total purity is calculated as the weighted average of the purity over all clusters, or formally as: $\sum_{i=1}^{k} \frac{\left|D_{i}\right|}{|D|} \cdot \operatorname{purity}\left(D_{i}\right)$. A greater value for purity means that the computer algorithm has done a better job of putting documents that belong together, as determined by human judgment, in the same cluster.

\section{Conclusion and Future Research}

In this paper, we reviewed how information from WordNet can be used to build a similarity graph. The graph shows the strength of the relationship between words and phrases from the English language. We showed how to use the graph to cluster documents. We use part of the Reuters- 21578 benchmark to fine-tune the algorithm. We showed that using the similarity graph leads to improved clustering as measure by precision, recall, f-score, and purity. We also showed that the fine-tuned algorithm improves these results even further and also gives us improved results on the entropy measure as compare to the cosine similarity algorithm.

One area for future research is using an extended version of the similarity graph that contains information from Wikipedia [37] to perform document clustering. One challenge in this area is that the extended graph is relatively big (more than $10 \mathrm{~GB}$ ) and computing the distance between documents can be computationally expensive. Another area for future research is to consider the order of the terms in the document. For example, the semantic similarity between documents that contain similar terms should be higher if the terms appear are in the same order.

\section{References}

[1] M. Agosti and F. Crestani, Automatic authoring and construction of hypertext for information retrieval, ACM Multimedia Systems 15(24) (1995).

[2] M. Agosti, F. Crestani, G. Gradenigo and P. Mattiello, An approach to conceptual modeling of IR auxiliary data, in IEEE International Conference on Computer and Communications, 1990.

[3] L. Burnard, Reference Guide for the British National Corpus (XML Edition). http:// www.natcorp.ox.ac.uk, 2007.

[4] P. Cohen and R. Kjeldsen, Information retrieval by constrained spreading activation on sematic networks, in Information Processing and Management, 1987, pp. 255-268. 
[5] R. Collobert and J. Weston, A unified architecture for natural language processing: Deep neural networks with multitask learning, in Twenty-Fifth International Conference on Machine Learning, 2008.

[6] F. Crestani, Application of spreading activation techniques in information retrieval, Artificial Intelligence Review 11(6) (1997) 453-482.

[7] Croft, User-specified domain knowledge for document retrieval, in Ninth Annual International ACM Conference on Research and Development in Information Retrieval, 1986, pp. 201-206.

[8] S. Deerwester, S. T. Dumais, G. W. Furnas, T. K. Landauer and R. Harshman, Indexing by latent semantic analysis, Journal of the Society for Information Science 41(6) (1990) 391-407.

[9] C. Fox, Lexical analysis and stoplists, in Information Retrieval: Data Structures and Algorithms, 1992, pp. 102-130.

[10] W. Frakes, Stemming algorithms, in Information Retrieval: Data Structures and Algorithms, 1992, pp. 131-160.

[11] T. Gruber, Collective knowledge systems: Where the social web meets the semantic, Web Journal of Web Semantics, 2008.

[12] R. V. Guha, R. McCool and E. Miller, Semantic search, in Twelfth International World Wide Web Conference, 2003, pp. 700-709.

[13] A. M. Harbourt, E. Syed, W. T. Hole and L. C. Kingsland, The ranking algorithm of the coach browser for the UMLS metathesaurus, in Seventeenth Annual Symposium on Computer Applications in Medical Care, 1993, pp. 720-724.

[14] W. R. Hersh and R. A. Greenes, SAPHIRE: An information retrieval system featuring concept matching, automatic indexing, probabilistic retrieval, and hierarchical relationships, in Computers and Biomedical Research, 1990, pp. 410-425.

[15] W. R. Hersh, D. D. Hickam and T. J. Leone, Words, concepts, or both: Optimal indexing units for automated information retrieval, in Sixteenth Annual Symposium on Computer Applications in Medical Care, 1992, pp. 644-648.

[16] E. H. Hovy, L. Gerber, U. Hermjakob, M. Junk and C. Y. Lin, Question answering in webclopedia, in TREC-9 Conference, 2000.

[17] K. Jarvelin, J. Keklinen and T. Niemi, ExpansionTool: Concept-based query expansion and construction, 2001, pp. 231-255.

[18] G. Jeh and J. Widom, SimRank: A measure of structural-context similarity, in Proceedings of the Eighth ACM SIGKDD International Conference on Knowledge Discovery and Data Mining, 2002, pp. 538-543.

[19] S. Jones, Thesaurus data model for an intelligent retrieval system, Journal of Information Science 19(1) (1993) 167-178.

[20] A. Kiryakov, B. Popov, I. Terziev, D. Manov and D. Ognyanoff, Semantic annotation, indexing, and retrieval, Journal of Web Semantics 2(1) (2004) 49-79.

[21] R. Knappe, H. Bulskov and T. Andreasen, Similarity graphs, in Fourteenth International Symposium on Foundations of Intelligent Systems, 2003.

[22] T. K. Landauer, P. Foltz and D. Laham, Introduction to latent semantic analysis, in Discourse Processes, 1998, pp. 259-284.

[23] J. B. MacQueen, Some methods for classification and analysis of multivariate observations, in Proceedings of Fifth Berkeley Symposium on Mathematical Statistics and Probability, 1967, pp. 281-297.

[24] M. F. Porter, An algorithm for suffix stripping, Readings in Information Retrieval, 1997, pp. 313-316.

[25] G. A. Miller, WordNet: A lexical database for English, Commun. ACM 38(11) (1995) $39-41$. 
[26] D. Moldovan, S. Harabagiu, M. Pasca, R. Mihalcea, R. Goodrum and R. Girju, LASSO: A tool for surfing the answer net, in Text Retrieval Conference (TREC-8), 1999.

[27] C. Paice, A thesaural model of information retrieval, Information Processing and Management 27(1) (1991) 433-447.

[28] B. Popov, A. Kiryakov, D. D. Ognyanoff, D. Manov and A. Kirilov. KIM - A semantic platform for information extraction and retrieval, Journal of Natural Language Engineering 10(3) (2004) 375-392.

[29] P. Resnik, Using information content to evaluate semantic similarity in a taxonomy, in International Joint Conference on Artificial Intelligence, 1995, pp. 448-453.

[30] L. Rau, Knowledge organization and access in a conceptual information system, Information Processing and Management 23(4) (1987) 269-283.

[31] S. S. Luke, L. Spector and D. Rager, Ontology-based knowledge discovery on the world wide web, in Internet-Based Information Systems: Papers from the AAAI Workshop, 1996, pp. 96-102.

[32] M. Sanderson, Word sense disambiguation and information retrieval, in Seventeenth Annual International ACM SIGIR Conference on Research and Development in Information Retrieval, 1994.

[33] P. Shoval, Expert consultation system for a retrieval database with semantic network of concepts, in Fourth Annual International ACM SIGIR Conference on Information Storage and Retrieval: Theoretical Issues in Information Retrieval, 1981, pp. 145-149.

[34] Simone Paolo Ponzetto and Michael Strube, Deriving a large scale taxonomy from wikipedia, in 22nd International Conference on Artificial Intelligence, 2007.

[35] B. Spell, Java API for WordNet Searching (JAWS), http://lyle.smu.edu/tspell/jaws/ index.html, 2009.

[36] K. Srihari, W. Li and X. Li, Information extraction supported question answering, in Advances in Open Domain Question Answering, 2004.

[37] L. Stanchev, Creating a phrase similarity graph from wikipedia, in Eighth IEEE International Conference on Semantic Computing, 2014.

[38] L. Stanchev, Creating a similarity graph from wordNet, in Fourth International Conference on Web Intelligence, Mining and Semantics, 2014.

[39] L. Stanchev, Measuring the strength of the semantic relationship between words, International Journal on Artificial Intelligence Tools, 2015.

[40] L. Stanchev, Semantic document clustering using a similarity graph, in Tenth IEEE International Conference on Semantic Computing, 2016, pp. 1-8.

[41] J. Turian, L. Ratinov and Y. Bengio, Word representations: A simple and general method for semi-supervised learning, in 48th Annual Meeting of the Association for Computational Linguistics, 2010, pp. 384-394.

[42] Y. Yang and C. G. Chute, Words or concepts: The features of indexing units and their optimal use in information retrieval, in Seventeenth Annual Symposium on Computer Applications in Medical Care, 1993, pp. 685-68. 